\title{
P2X7 receptor involvement in COVID-19 disease
}

\author{
Peter Illes ${ }^{1,2 *}$ \\ ${ }^{1}$ Rudolf Boehm Institute for Pharmacology and Toxicology, University of Leipzig, 04107 Leipzig, Germany \\ ${ }^{2}$ International Collaborative Center on Big Science Plan for Purinergic Signaling, Chengdu University of Traditional Chinese \\ Medicine, Chengdu 610000, Sichuan, China
}

*Correspondence: Peter Illes, Rudolf Boehm Institute for Pharmacology and Toxicology, University of Leipzig, 04107 Leipzig, Germany; International Collaborative Center on Big Science Plan for Purinergic Signaling, Chengdu University of Traditional Chinese Medicine, Chengdu 610000, Sichuan, China. peter.illes@medizin.uni-leipzig.de

Academic Editor: Rafael Franco, Universidad de Barcelona, Spain

Received: February 10, 2021 Accepted: February 26, 2021 Published: August 5, 2021

Cite this article: Illes P. P2X7 receptor involvement in COVID-19 disease. Explor Neuroprot Ther. 2021;1:7-9. https://doi. org/10.37349/ent.2021.00002

\section{Keywords}

COVID-19, SARS-CoV-2, P2X7 receptor, angiotensin-converting enzyme 2, cytokine storm, central nervous system complications

Coronavirus disease 2019 (COVID-19) is a pandemic which has spread during the last year all over the world. This disease causes serious health consequences, especially but not exclusively in aged patients. Because of the lockdowns introduced by governments to prevent the distribution of COVID-19, it also profoundly interfered with undisturbed economic development. It has been revealed that contamination might be originally due to human contact with bats; severe acute respiratory syndrome coronavirus 2 (SARS-CoV-2, the causative virus of COVID19) appears to be closely related to other bat-hosted coronaviruses responsible for the potentially fatal acute respiratory distress syndrome (ARDS) [1]. SARS-CoV-2 enters human cells by using viral spike proteins for binding to angiotensin-converting enzyme (ACE)2. In addition, as a pre-requisite for crossing the cell membrane of their target cells (e.g., the bronchial/alveolar epithelium), a complex of the ACE2 receptor with a transmembrane serine protease 2 (TMPRSS2) is generated which allows fusion of the plasma membrane with the viral envelope. Pneumonia, the main cause of morbidity and mortality in COVID-19 patients is thought to be due to uncontrolled, virus-mediated direct activation of lung macrophages in a process resembling the macrophage activation syndrome (MAS), observed in some rheumatological diseases [2]. COVID-19 patients show massive infiltration by inflammatory cells (neutrophils and monocytes/ macrophages) of their lungs and increased blood levels of inflammatory cytokines and chemokines. This so called "cytokine storm" syndrome then causes severe pneumonia that fulfills most criteria of an ARDS (see above) in which severe hypoxemia is associated with near normal respiratory system compliance. In addition to cytokines/chemokines also the release of reactive oxygen species (ROS), secretion of vascular endothelial growth factor (VEGF), and T lymphocyte exhaustion are causally involved in pneumonia. VEGF causes pulmonary edema and thromboembolic complications. 
P2X7 receptors (P2X7Rs) are members of the ligand-gated P2XR family [3]. In contrast to other P2XRs they are activated by high micromolar concentrations of ATP, released during cell-damaging conditions into the extracellular space. P2X7Rs are located at immune cells of the periphery and microglia, the resident macrophages of the central nervous system (CNS). P2X7Rs on their opening permit the influx of $\mathrm{Na}^{+} / \mathrm{Ca}^{2+}$ and the outflux of $\mathrm{K}^{+}$. However, on long-lasting activation with ATP they were assumed for many years to generate membrane pores permeable to large organic cations such as the fluorescent dye Yo-Pro-1. More recently, it was concluded that ATP does not convert the P2X7 ionophore into a membrane pore, but recruits an accessory protein, the pannexin-1 channel, or even that P2X7R channels allow the passage of large cationic molecules immediately from their initial activation, but at a much slower pace than that of the smaller cations.

P2X7Rs are major drivers of inflammation. Macrophages/microglia are equipped with a battery of pattern recognition receptors that detect pathogen-associated molecular patterns (PAMPs) such as lipopolysaccharide (LPS) from bacterial infection or danger-associated molecular patterns (DAMPs), such

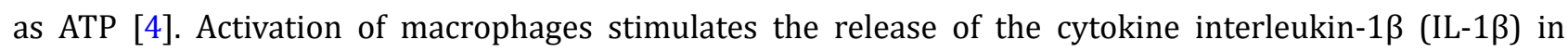
a two-step process: the first being the stimulation of toll-like receptor 4 by LPS and the second being the ATP-dependent stimulation of P2X7Rs, promoting nucleotide-binding, leucine-rich repeat, pyrin domain containing protein 3 (NLRP3) inflammasome-mediated caspase-1 activation and subsequent secretion of IL-1 $\beta$. Caspase- 1 generates IL-1 $\beta$ by splitting its precursor pro-IL-1 $\beta$.

Novel evidence indicates that the SARS-CoV-2-released mediators and ATP through P2X7R stimulation share common intracellular pathways, upregulating cytosolic DAMP receptors [5]. In both cases the NLRP3 inflammasome is centrally involved in events leading eventually to the COVID-19-characteristic "cytokine storm". NLRP3 activation during COVID-19 infection is usually triggered by the octapeptide angiotensin (Ang) II which binds to the angiotensin II type 1 receptor $\left(\mathrm{AT}_{1} \mathrm{R}\right)$, leading to vessel contraction and increased blood pressure. ACE1 generates the biologically active Ang II from its inactive precursor Ang I. By contrast ACE2 degrades Ang II to Ang 1-7 which counteracts the effect of Ang II via the production of the vasodilatory and cardioprotective nitrogen monoxide (NO). When COVID-19 has been bound to its receptor ACE2, this enzyme is no longer available for occupation by Ang II, and in consequence the beneficiary cardiovascular effect of NO is lost. As already mentioned above, inflammatory responses may also cause endothelial damage and consequently increased production of thrombin by inflammatory cytokines.

Pulmonary, renal and cardiovascular symptoms of COVID-19 are generally appreciated but more recently neurological symptoms were also reported $[5,6]$. Various routes may be used by SARS-CoV-2 to enter the CNS such as (1) infected leukocytes to carry their virus load through the damaged blood-brain-barrier; (2) retrograde transport by using olfactory sensory nerves projecting to the nasal cavity. Neuroinflammation by COVID-19 may worsen existing neurodegenerative diseases (Alzheimer's and Parkinson's Disease) but also neuropsychiatric disorders (major depression, bipolar disorder, anxiety). The idea is that the cytokine storm induced by viral infection could promote neuroinflammation and neuronal death.

The numerous similarities between COVID-19 symptoms and those induced by P2X7R activation make it likely that low molecular weight P2X7R antagonists are possible therapeutic agents to treat the viral disease [2]. An obvious marker to monitor the efficiency of the treatment regime would be blood IL-1 $\beta$ levels. COVID-19 patients with rapidly evolving ARDS would be especially appropriate individuals to use pharmacological antagonists of P2X7R to treat this life-threatening condition.

\section{Abbreviations}

ACE: angiotensin-converting enzyme

Ang: angiotensin

ARDS: acute respiratory distress syndrome

COVID-19: coronavirus disease 2019

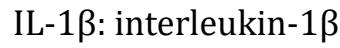

NLRP3: nucleotide-binding, leucine-rich repeat, pyrin domain containing protein 3 
P2X7Rs: P2X7 receptors

SARS-CoV-2: severe acute respiratory syndrome coronavirus 2

\section{Declarations}

\section{Author contributions}

The author contributed solely to the work.

\section{Conflicts of interest}

The author declares that there are no conflicts of interest.

\section{Ethical approval}

Not applicable.

\section{Consent to participate}

Not applicable.

\section{Consent to publication}

Not applicable.

Availability of data and materials

Not applicable.

\section{Funding}

Not applicable.

\section{Copyright}

(C) The Author(s) 2021.

\section{References}

1. Dos Anjos F, Simoes JLB, Assmann CE, Carvalho FB, Bagatini MD. Potential therapeutic role of purinergic receptors in cardiovascular disease mediated by SARS-CoV-2. J Immunol Res. 2020;2020:8632048.

2. Di Virgilio F, Tang Y, Sarti AC, Rossato M. A rationale for targeting the P2X7 receptor in Coronavirus disease 19. Br J Pharmacol. 2020;177:4990-4.

3. Illes P, Rubini P, Ulrich $\mathrm{H}$, Zhao Y, Tang Y. Regulation of microglial functions by purinergic mechanisms in the healthy and diseased CNS. Cells. 2020;9:5.

4. Illes P, Verkhratsky A, Tang Y. Pathological ATPergic signaling in major depression and bipolar disorder. Front Mol Neurosci. 2020;12:331.

5. Ribeiro DE, Oliveira-Giacomelli A, Glaser T, Arnaud-Sampaio VF, Andrejew R, Dieckmann L, et al. Hyperactivation of P2X7 receptors as a culprit of COVID-19 neuropathology. Mol Psychiatry. 2021;26:1044-59.

6. Simoes JLB, Bagatini MD. Purinergic signaling of ATP in COVID-19 associated Guillain-Barre syndrome. J Neuroimmune Pharmacol. 2021;16:48-58. 